\title{
Evaluation of a ln tan integral arising in quantum field theory
}

\author{
Mark W. Coffey \\ Department of Physics \\ Colorado School of Mines \\ Golden, CO 80401
}

(Received

2007)

August 27, 2007

\begin{abstract}
We analytically evaluate a dilogarithmic integral that is prototypical of volumes of ideal tetrahedra in hyperbolic geometry. We additionally obtain new representations of the Clausen function $\mathrm{Cl}_{2}$ and the Catalan constant $G=\mathrm{Cl}_{2}(\pi / 2)$, as well as new relations between sine and Clausen function values.
\end{abstract}

\section{Key words and phrases}

Clausen function, trigamma function, polygamma function, dilogarithm function, Hurwitz zeta function

\section{AMS classification numbers}

33B30, 33B15, 11M35 


\section{Introduction and statement of results}

In this paper, we provide a closed form evaluation of the integral

$$
I_{7} \equiv \frac{24}{7 \sqrt{7}} \int_{\pi / 3}^{\pi / 2} \ln \left|\frac{\tan t+\sqrt{7}}{\tan t-\sqrt{7}}\right| d t
$$

in terms of elementary mathematical constants and values of the Clausen function $\mathrm{Cl}_{2}$. This and related integrals originate in hyperbolic geometry and quantum field theory [7, 14]. The integral $I_{7}$ arises in the analysis of the volume of ideal tetrahedra in hyperbolic space and is the simplest of 998 empirically investigated cases wherein the volume of a hyperbolic knot complement appears to be expressible in terms of a Dirichlet L series [5]. In particular, Borwein and Broadhurst have conjectured that $I_{7} \stackrel{?}{=} L_{-7}(2)=\sum_{n=0}^{\infty}\left[\frac{1}{(7 n+1)^{2}}+\frac{1}{(7 n+2)^{2}}-\frac{1}{(7 n+3)^{2}}+\frac{1}{(7 n+4)^{2}}-\frac{1}{(7 n+5)^{2}}-\frac{1}{(7 n+6)^{2}}\right]$.

The ? here indicates numerical verification has been performed but that no proof exists.

The integral (1.1) has been much discussed of late 4], and in fact it provides the opening equation of a very recent book [3]. The evaluation of $I_{7}$ in the specific form of Eq. (1.2) remains to be completed-our evaluation is in terms of a certain other combination of Clausen function values. In addition to the Clausen value relations conjectured in Ref. [5], our work suggests further, presumably kindred, relations. Moreover, our work may provide a means of making progress in the other 997 cases linking a knot complement volume to an L series. The equality of expressions such as Eqs. (1.1) and (1.2) would tightly link topics in hyperbolic geometry with analytic 
number theory.

In the rest of this Introduction, we present two Lemmas, state our main result, and provide some background relations on the particular Clausen function $\mathrm{Cl}_{2}$.

It seems worth while to note some of the numerous alternative expressions for the value $L_{-7}(2)$ and its companion Clausen value combination. We have

Lemma 1. Let $\psi^{\prime}$ be the trigamma function and $\zeta(s, a)$ the Hurwitz zeta function (e.g, [1]). (a) We have

$$
\begin{gathered}
L_{-7}(2)=\frac{1}{49}\left[\psi^{\prime}\left(\frac{1}{7}\right)+\psi^{\prime}\left(\frac{2}{7}\right)-\psi^{\prime}\left(\frac{3}{7}\right)+\psi^{\prime}\left(\frac{4}{7}\right)-\psi^{\prime}\left(\frac{5}{7}\right)-\psi^{\prime}\left(\frac{6}{7}\right)\right] \\
=\frac{1}{49}\left[2\left(\psi^{\prime}\left(\frac{1}{7}\right)+\psi^{\prime}\left(\frac{2}{7}\right)-\psi^{\prime}\left(\frac{3}{7}\right)\right)-\pi^{2}\left(\csc ^{2} \frac{\pi}{7}+\csc ^{2} \frac{2 \pi}{7}-\csc ^{2} \frac{3 \pi}{7}\right)\right] \\
=\frac{2}{49}\left[\psi^{\prime}\left(\frac{1}{7}\right)+\psi^{\prime}\left(\frac{2}{7}\right)-\psi^{\prime}\left(\frac{3}{7}\right)+\left(\csc ^{2} \frac{3 \pi}{7}-4\right) \pi^{2}\right] \\
=\frac{1}{49}\left[\zeta\left(2, \frac{1}{7}\right)+\zeta\left(2, \frac{2}{7}\right)-\zeta\left(2, \frac{3}{7}\right)+\zeta\left(2, \frac{4}{7}\right)-\zeta\left(2, \frac{5}{7}\right)-\zeta\left(2, \frac{6}{7}\right)\right] \\
=-\int_{0}^{1} \frac{\left(1+u-u^{2}+u^{3}-u^{4}-u^{5}\right)}{\left(1-u^{7}\right)} \ln u d u \\
=-\int_{0}^{1} \frac{\left(1+2 u+u^{2}+2 u^{3}+u^{4}\right)}{\left(1+u+u^{2}+u^{3}+u^{4}+u^{5}+u^{6}\right)} \ln u d u \\
=1-\int_{0}^{1} \frac{u\left(1+u-u^{4}-u^{5}\right)}{\left(1+u+u^{2}+u^{3}+u^{4}+u^{5}+u^{6}\right)} \ln u d u
\end{gathered}
$$

(b) We have

$$
\begin{gathered}
\mathrm{Cl}_{2}\left(\frac{2 \pi}{7}\right)+\mathrm{Cl}_{2}\left(\frac{4 \pi}{7}\right)-\mathrm{Cl}_{2}\left(\frac{6 \pi}{7}\right)=\frac{1}{56 \sqrt{7}}\left\{8\left[\psi^{\prime}\left(\frac{1}{7}\right)+\psi^{\prime}\left(\frac{2}{7}\right)-\psi^{\prime}\left(\frac{3}{7}\right)\right]\right. \\
\left.\pi^{2}\left(-\csc ^{2} \frac{\pi}{14}+2 \csc ^{2} \frac{3 \pi}{7}++\csc ^{2} \frac{\pi}{7}+\csc ^{2} \frac{3 \pi}{14}+\csc ^{2} \frac{2 \pi}{7}+\csc ^{2} \frac{5 \pi}{14}-\csc ^{2} \frac{4 \pi}{7}\right)\right\} .
\end{gathered}
$$


In Proposition 1 we make use of some of the representations for $\mathrm{Cl}_{2}$ given in the following

Lemma 2. Let $a$ be a real number and set

$$
\theta=\cos ^{-1}\left(\frac{1-a^{2}}{1+a^{2}}\right)
$$

Then we have (a)

$$
\mathrm{Cl}_{2}(\theta)=\int_{a}^{\infty} \ln \left(\frac{u+a}{u-a}\right) \frac{d u}{1+u^{2}}
$$

Let $H_{n}$ be the $n$th harmonic number, $\gamma=-\psi(1)$ the Euler constant, and $\psi$ the digamma function. Then (b)

$$
\begin{gathered}
\mathrm{Cl}_{2}(\theta)=2 \cot ^{-1} a \ln 2+\frac{1}{a} \sum_{j=1}^{\infty} \frac{(-1)^{j}}{a^{2 j}} \frac{H_{j}}{(2 j+1)} \\
=\cot ^{-1} a(2 \ln 2+\gamma-2)+\frac{1}{a}(2-\gamma)-\frac{1}{a} \ln \left(1+\frac{1}{a^{2}}\right)+\frac{1}{a} \sum_{j=1}^{\infty} \frac{(-1)^{j}}{a^{2 j}} \frac{\psi(j)}{(2 j+1)} .
\end{gathered}
$$

(c) We have the integral representation

$$
\mathrm{Cl}_{2}(\theta)=2 \cot ^{-1} a \ln 2+2 a \int_{1}^{\infty} \frac{\left[y \cot ^{-1} a y-\cot ^{-1} a\right]}{\left(1-y^{2}\right)} \frac{d y}{y} .
$$

As an immediate consequence of Lemma $2(\mathrm{~b})$ at $a=1$ we obtain a harmonic number series representation for the Catalan constant $G=\mathrm{Cl}_{2}(\pi / 2)=1-1 / 3^{2}+$ $1 / 5^{2}-1 / 7^{2}+\ldots \simeq 0.9159655941:$

Corollary 1. We have

$$
G=\frac{\pi}{2} \ln 2+\sum_{j=1}^{\infty} \frac{(-1)^{j}}{(2 j+1)} H_{j}
$$

This expression has been previously obtained in the very recent Ref. [6]. 
Let $\theta_{ \pm}= \pm \tan ^{-1}(\sqrt{7} / 3)$

$$
r_{73} \equiv \frac{\sqrt{7}+\sqrt{3}}{\sqrt{7}-\sqrt{3}}, \quad \omega_{ \pm}=\tan ^{-1}\left(\frac{r_{73} \sin \theta_{ \pm}}{1-r_{73} \cos \theta_{ \pm}}\right)
$$

so that

$$
\begin{gathered}
\omega_{+}=-\cot ^{-1}(2 \sqrt{3}-\sqrt{7})=\tan ^{-1} \sqrt{7}-\frac{2 \pi}{3} \\
\omega_{-}=-\omega_{+}=\tan ^{-1}\left(\frac{2 \sqrt{3}-\sqrt{7}}{5}\right), \quad \text { and } \quad \omega_{+}-\omega_{-}=-2 \omega_{+}=-2 \cot ^{-1}(2 \sqrt{3}-\sqrt{7}) .
\end{gathered}
$$

Proposition 1. We have

$$
I_{7}=\frac{24}{7 \sqrt{7}}\left\{\mathrm{Cl}_{2}\left(\theta_{+}\right)+\frac{1}{2}\left[\mathrm{Cl}_{2}\left(2 \omega_{+}\right)-\mathrm{Cl}_{2}\left(2 \omega_{+}+2 \theta_{+}\right)\right]\right\} .
$$

The Clausen function $\mathrm{Cl}_{2}$ can be defined by (e.g., [13])

$$
\begin{aligned}
\mathrm{Cl}_{2}(\theta) & \equiv-\int_{0}^{\theta} \ln \left(2 \sin \frac{t}{2}\right) d t=\int_{0}^{1} \tan ^{-1}\left(\frac{x \sin \theta}{1-x \cos \theta}\right) \frac{d x}{x} \\
& =-\sin \theta \int_{0}^{1} \frac{\ln x}{x^{2}-2 x \cos \theta+1} d x=\sum_{n=1}^{\infty} \frac{\sin (n \theta)}{n^{2}} .
\end{aligned}
$$

When $\theta$ is a rational multiple of $\pi$ it is known that $\mathrm{Cl}_{2}(\theta)$ may be written in terms of the trigamma and sine functions [10, 8]. As we shall find useful, the imaginary part of the dilogarithm function $\mathrm{Li}_{2}$ of complex argument may be written in terms of $\mathrm{Cl}_{2}$ :

$$
\operatorname{Im} \operatorname{Li}_{2}\left(r e^{i \theta}\right)=\omega \ln r+\frac{1}{2}\left[\mathrm{Cl}_{2}(2 \omega)-\mathrm{Cl}_{2}(2 \omega+2 \theta)+\mathrm{Cl}_{2}(2 \theta)\right]
$$

where

$$
\omega=\tan ^{-1}\left(\frac{r \sin \theta}{1-r \cos \theta}\right)
$$


Besides being periodic, $\mathrm{Cl}_{2}(\theta)=\mathrm{Cl}_{2}(\theta+2 \pi)$, the Clausen function satisfies the duplication formula

$$
\frac{1}{2} \mathrm{Cl}_{2}(2 \theta)=\mathrm{Cl}_{2}(\theta)-\mathrm{Cl}_{2}(\pi-\theta) .
$$

Further properties of $\mathrm{Cl}_{2}$ are given in [12] and [13] and Ref. [15] gives a very recent but necessarily incomplete review of the dilogarithm function with complex argument.

We next provide the proofs of Lemmas 1 and 2 and Proposition 1 and finish with a discussion with further analytic results. Our proof of Proposition 1 shows how to make a ready generalization of the integral $I_{7}$.

\section{Proofs of Lemmas 1 and 2, and expressions for the Catalan constant}

For Eqs. (1.3) and (1.4) of Lemma 1, we repeatedly apply the relation [9] (p. 944) between the specific Hurwitz zeta function $\zeta(2, a)$ and the trigamma function contained in

$$
\sum_{j=0}^{\infty} \frac{1}{(7 j+p)^{q}}=\frac{1}{7^{q}} \zeta\left(q, \frac{p}{7}\right)=\frac{(-1)^{q}}{7^{q}} \frac{1}{(q-1) !} \psi^{(q-1)}\left(\frac{p}{7}\right),
$$

and use the reflection formula satisfied by the trigamma function

$$
\psi^{\prime}(1-x)=-\psi^{\prime}(x)+\pi^{2} \csc ^{2} \pi x .
$$

Equation (1.3c) follows from (1.3b) by the use of the identity

$$
\csc ^{2} \frac{\pi}{7}+\csc ^{2} \frac{2 \pi}{7}+\csc ^{2} \frac{3 \pi}{7}=8
$$

For Eq. (1.5), we use the integral representation

$$
\zeta(s, a)=\frac{1}{\Gamma(s)} \int_{0}^{\infty} \frac{t^{s-1} e^{-(a-1) t}}{e^{t}-1} d t, \quad \operatorname{Re} s>1, \quad \operatorname{Re} a>0
$$


where $\Gamma$ is the Gamma function, and change variable to $u=e^{-t / 7}$.

For part (b) of Lemma 1, we first apply for $p$ even and $q$ odd, with $q \geq 3$ the formula [8] (p. 329)

$$
\mathrm{Cl}_{2}\left(\frac{p \pi}{q}\right)=-\frac{1}{4 q^{2}} \sum_{k=1}^{q-1}\left[\psi^{\prime}\left(1-\frac{k}{2 q}\right)+\psi^{\prime}\left(\frac{1}{2}-\frac{k}{2 q}\right)\right] \sin k \frac{p}{q} \pi .
$$

We then obtain

$$
\begin{gathered}
\mathrm{Cl}_{2}\left(\frac{2 \pi}{7}\right)+\mathrm{Cl}_{2}\left(\frac{4 \pi}{7}\right)-\mathrm{Cl}_{2}\left(\frac{6 \pi}{7}\right) \\
=\frac{1}{56 \sqrt{7}}\left[\psi^{\prime}\left(\frac{1}{14}\right)+\psi^{\prime}\left(\frac{1}{7}\right)-\psi^{\prime}\left(\frac{3}{14}\right)+\psi^{\prime}\left(\frac{2}{7}\right)-\psi^{\prime}\left(\frac{5}{14}\right)-\psi^{\prime}\left(\frac{3}{7}\right)\right. \\
\left.+\psi^{\prime}\left(\frac{4}{7}\right)+\psi^{\prime}\left(\frac{9}{14}\right)-\psi^{\prime}\left(\frac{5}{7}\right)+\psi^{\prime}\left(\frac{11}{14}\right)-\psi^{\prime}\left(\frac{6}{7}\right)-\psi^{\prime}\left(\frac{13}{14}\right)\right],
\end{gathered}
$$

wherein we used the identity

$$
\sin \frac{2 x \pi}{7}+\sin \frac{4 x \pi}{7}-\sin \frac{6 x \pi}{7}= \pm \frac{\sqrt{7}}{2}
$$

for $x=1, \ldots, 6$ and the - sign holds on the right side for $x=3,5$, and 6 .

In regard to Eq. (2.7), we observe that $\pm \sin (2 \pi / 7), \pm \sin (4 \pi / 7)$, and $\pm \sin (6 \pi / 7)$ are the nonzero roots of the Chebyshev polynomial $T_{7}(x)$. Indeed, if we write the cubic polynomials

$$
p_{1}(x)=\left(x-\sin \frac{2 \pi}{7}\right)\left(x-\sin \frac{4 \pi}{7}\right)\left(x+\sin \frac{6 \pi}{7}\right)=x^{3}-\frac{\sqrt{7}}{2} x^{2}+\frac{\sqrt{7}}{8},
$$

and

$$
p_{2}(x)=\left(x-\sin \frac{6 \pi}{7}\right)\left(x+\sin \frac{2 \pi}{7}\right)\left(x+\sin \frac{4 \pi}{7}\right)=x^{3}+\frac{\sqrt{7}}{2} x^{2}-\frac{\sqrt{7}}{8}
$$

we then have the factorization $p_{1}(x) p_{2}(x)=T_{7}(x) / 64 x$. 
We next repeatedly apply the duplication formula

$$
2 \psi^{\prime}(2 x)=\frac{1}{2}\left[\psi^{\prime}(x)+\psi^{\prime}\left(x+\frac{1}{2}\right)\right]
$$

and the reflection formula (2.2) in order to reduce the dozen trigamma values in Eq. (2.6) to only three independent function values. In particular, we have the relations

$$
\begin{aligned}
& \psi^{\prime}\left(\frac{1}{14}\right)=4 \psi^{\prime}\left(\frac{1}{7}\right)+\psi^{\prime}\left(\frac{3}{7}\right)-\pi^{2} \csc ^{2} \frac{4 \pi}{7} \\
& \psi^{\prime}\left(\frac{3}{14}\right)=4 \psi^{\prime}\left(\frac{3}{7}\right)+\psi^{\prime}\left(\frac{2}{7}\right)-\pi^{2} \csc ^{2} \frac{2 \pi}{7}
\end{aligned}
$$

and

$$
\psi^{\prime}\left(\frac{5}{14}\right)=-4 \psi^{\prime}\left(\frac{2}{7}\right)+\psi^{\prime}\left(\frac{1}{7}\right)-\pi^{2} \csc ^{2} \frac{\pi}{7}+4 \pi^{2} \csc ^{2} \frac{2 \pi}{7}
$$

and such substitutions give Eq. (1.6).

Equation (1.6) could be further rewritten with Eq. (2.3) and/or the identity

$$
\csc ^{2} \frac{\pi}{14}+\csc ^{2} \frac{\pi}{7}+\csc ^{2} \frac{3 \pi}{14}+\csc ^{2} \frac{2 \pi}{7}+\csc ^{2} \frac{5 \pi}{14}+\csc ^{2} \frac{3 \pi}{7}=32
$$

that is another case of the relation $\sum_{j=1}^{[(n-1) / 2]} \csc ^{2}\left(\frac{j \pi}{n}\right)=\left(n^{2}-1\right) / 6-\left[1+(-1)^{n}\right] / 4$ (e.g., [11], p. 260). Indeed, there are at most two independent trigamma values involved in either part (a) or (b) of Lemma 1. As follows from the multiplication formula for the polygamma functions, we have [1] (p. 260)

$$
\psi^{\prime}(m x)=\frac{1}{m^{2}} \sum_{k=0}^{m-1} \psi^{\prime}\left(x+\frac{k}{m}\right),
$$

so that we have the additional relation

$$
\zeta(2)=\psi^{\prime}(1)=\frac{1}{49} \sum_{k=0}^{6} \psi^{\prime}\left(\frac{k+1}{7}\right) .
$$


For part (a) of Lemma 2, we consider the integrals

$$
\int_{a}^{\infty} \ln ^{n}\left(\frac{u+a}{u-a}\right) \frac{d u}{1+u^{2}}=\frac{2 a}{\left(1+a^{2}\right)} \int_{1}^{\infty} \frac{\ln ^{n} v d v}{v^{2}+2\left(\frac{a^{2}-1}{a^{2}+1}\right) v+1}
$$

where we changed variable to $v=(u+a) /(u-a)$. With the further change of variable $y=1 / v$ and use of the definition (1.7) for $\theta$ we have

$$
\int_{a}^{\infty} \ln ^{n}\left(\frac{u+a}{u-a}\right) \frac{d u}{1+u^{2}}=\frac{2 a(-1)^{n}}{\left(1+a^{2}\right)} \int_{0}^{1} \frac{\ln ^{n} y d y}{y^{2}-2 \cos \theta y+1}
$$

Therefore, by the integral representation of $\mathrm{Cl}_{2}$ (1.14), at $n=1$ we obtain Eq. (1.8).

For part (b), we start with the result of part (a), and change variable to $y=1 / u$ to obtain

$$
\mathrm{Cl}_{2}(\theta)=\int_{0}^{1 / a} \ln \left(\frac{1+a y}{1-a y}\right) \frac{d y}{1+y^{2}} .
$$

We then make use of the relation [9] (p. 1041)

$$
\ln \left(\frac{1+z}{1-z}\right)=2 z_{2} F_{1}\left(\frac{1}{2}, 1 ; \frac{3}{2} ; z^{2}\right)
$$

where ${ }_{2} F_{1}$ is the Gauss hypergeometric function. Changing variable again to $w=a^{2} y^{2}$ and expanding the factor $\left(1+y^{2}\right)^{-1}$ as a geometric series we have

$$
\mathrm{Cl}_{2}(\theta)=\frac{1}{2 a} \sum_{j=0}^{\infty} \frac{(-1)^{j}}{a^{2 j}} \int_{0}^{1} w_{2}^{j} F_{1}\left(\frac{1}{2}, 1 ; \frac{3}{2} ; w\right) d w .
$$

Performing the integration term by term, we have

$$
\begin{gathered}
\int_{0}^{1} w_{2}^{j} F_{1}\left(\frac{1}{2}, 1 ; \frac{3}{2} ; w\right) d w=\sum_{\ell=0}^{\infty} \frac{1}{2 \ell+1} \int_{0}^{1} w^{j+\ell} d w \\
=\sum_{\ell=0}^{\infty} \frac{1}{(2 \ell+1)(j+\ell+1)}=\frac{1}{(2 j+1)}[\psi(j+1)+\gamma+2 \ln 2],
\end{gathered}
$$


where the partial fractions form of the digamma function was used. Since

$$
\psi(j+1)+\gamma=H_{j}
$$

we have found the representation (1.9a). For Eq. (1.9b) we use the functional equation of the digamma function, $\psi(j+1)=\psi(j)+1 / j$. The absolute convergence of integrals such as in Eq. (2.9) justifies the interchange of integration and summation above and throughout this paper.

For Eq. (1.10), we use an integral representation of the digamma function [9] (p. 943) to write

$$
\begin{gathered}
\sum_{j=1}^{\infty} \frac{(-1)^{j}}{a^{2 j}} \frac{1}{2 j+1}[\psi(j+1)+\gamma]=\sum_{j=1}^{\infty} \frac{(-1)^{j}}{a^{2 j}} \frac{1}{2 j+1} \int_{0}^{1} \frac{\left(t^{j}-1\right)}{(t-1)} d t \\
=a \int_{0}^{1}\left[\cot ^{-1}\left(\frac{a}{\sqrt{t}}\right) \frac{1}{\sqrt{t}}-\cot ^{-1} a\right] d t
\end{gathered}
$$

where we interchanged summation and integration. With the change of variable $y=1 / \sqrt{t}$, we arrive at the integral representation (1.10).

Remarks on the Catalan constant. We have noted that Corollary 1 directly follows from Lemma 2(b). Another means to find the result (1.11) is to use the integral relation

$$
G=\frac{\pi}{2} \ln 2-\frac{1}{2} \int_{0}^{1} \frac{\ln (1+u)}{(1+u)} \frac{d u}{\sqrt{u}} .
$$

Then the use of the generating function

$$
-\frac{\ln (1+u)}{(1+u)}=\sum_{n=1}^{\infty}(-1)^{n} H_{n} u^{n},
$$

returns Eq. (1.11). 
Another form of the Catalan constant may be found by expanding in the integrand of Eq. (2.22) to write

$$
G=\frac{\pi}{2} \ln 2-\frac{1}{2} \sum_{j=0}^{\infty}(-1)^{j} \int_{0}^{1} \ln (1+u) u^{j-1 / 2} d u .
$$

Omitting the details, we determine

$$
G=-\frac{\pi}{4} \ln 2+\frac{1}{2} \sum_{j=0}^{\infty} \frac{(-1)^{j}}{(2 j+1)}\left[\psi\left(\frac{j}{2}+\frac{3}{4}\right)-\psi\left(\frac{j}{2}+\frac{1}{4}\right)\right]
$$

The sum in this equation may be re-expressed by applying the duplication formula for the digamma function.

With $\theta=\theta(a)$ defined as in Eq. (1.7), Eq. (1.8) may be rewritten as

$$
\mathrm{Cl}_{2}(\theta)=\cot ^{-1} a \ln a+2 \int_{a}^{\infty} \operatorname{coth}^{-1}\left(\frac{u}{a}\right) \frac{d u}{1+u^{2}}
$$

Then we obtain

$$
G=2 \int_{1}^{\infty} \frac{\operatorname{coth}^{-1} u}{1+u^{2}} d u
$$

We next have

Lemma 3. We have

$$
\begin{gathered}
G=-\frac{1}{4} \int_{0}^{1} \frac{\ln x / 2}{(1-x / 2)} \frac{d x}{\sqrt{1-x^{2}}} \\
=\frac{\pi}{4} \ln 2+\frac{\sqrt{\pi}}{4} \sum_{j=0}^{\infty} \frac{j !}{2^{j} \Gamma(j+3 / 2)}\left[\psi\left(j+\frac{3}{2}\right)-\psi(j+1)\right],
\end{gathered}
$$

where $2^{j} \Gamma(j+3 / 2)=\sqrt{\pi}(j+1 / 2)(2 j-1) !$ !, and

$$
G=\frac{\pi}{4} \ln 2+2 \int_{0}^{1} \frac{\sin ^{-1}(y / \sqrt{2}) d y}{(y+1) \sqrt{2-y^{2}}} .
$$


For obtaining Eq. (2.28a) we apply Eqs. (18) and (21) of Ref. [14]. For Eq. (2.28b) we expand the integrand factor $1-x / 2$ as geometric series, writing

$$
G=-\frac{1}{4} \sum_{j=0}^{\infty} \frac{1}{2^{j}} \int_{0}^{1} x^{j} \ln \left(\frac{x}{2}\right) \frac{d x}{\sqrt{1-x^{2}}}
$$

Performing the integral and using the series ([11], p. 151 or 174)

$$
\sum_{j=0}^{\infty} \frac{j !}{2^{j} \Gamma(j+3 / 2)}=\frac{1}{\Gamma(3 / 2)} \sum_{j=0}^{\infty} \frac{j !}{2^{j}(3 / 2)_{j}}=\sqrt{\pi}
$$

where $(a)_{j}$ is the Pochhammer symbol, gives Eq. (2.28b). The sum in the latter equation may be re-expressed by using the duplication formula of the digamma function. For Eq. (2.28c) we inserted an integral representation of $\psi$ into Eq. (2.28b).

By using an integral representation

$$
\operatorname{coth}^{-1} z=\frac{1}{z} \int_{0}^{1} \frac{d t}{1-t^{2} / z^{2}}
$$

and interchanging integrations we obtain from Eq. (2.26) with $\theta$ as in Eq. (1.7)

$$
\mathrm{Cl}_{2}(\theta)=\cot ^{-1} a \ln a+\left[\ln \left(a^{2}+1\right)-2 \ln a\right] \tan ^{-1} a-\int_{0}^{1} \frac{\ln \left(1-t^{2}\right)}{1+a^{2} t^{2}} d t
$$

giving

$$
G=\frac{\pi}{4} \ln 2-\int_{0}^{1} \frac{\ln \left(1-t^{2}\right)}{1+t^{2}} d t .
$$

This last equation may also be obtained by combining two formulas in Ref. [9] (p. 555). By expanding $\left(1+a^{2} t^{2}\right)^{-1}$ as a geometric series in Eq. (2.32) we obtain the series representation

$$
\mathrm{Cl}_{2}(\theta)=\cot ^{-1} a \ln a+\left[\ln \left(a^{2}+1\right)-2 \ln a\right] \tan ^{-1} a-\sum_{j=0}^{\infty}(-1)^{j} \frac{a^{2 j}}{(2 j+1)}\left[\psi\left(j+\frac{3}{2}\right)+\gamma\right] .
$$


We have also determined a BBP-type formula for the Catalan constant [2], as well as for a trilogarithmic constant. We have

Lemma 4. (a) We have the expression for $G=\mathrm{Cl}_{2}(\pi / 2)$

$$
G=\frac{1}{4} \sum_{j=0}^{\infty} \frac{1}{16^{j}}\left[\frac{4}{(8 j+1)^{2}}-\frac{2}{(8 j+4)^{2}}-\frac{1}{(8 j+5)^{2}}-\frac{1}{(8 j+6)^{2}}\right]-\frac{\pi^{2}}{32}+\frac{\pi}{8} \ln 2,
$$

$$
\operatorname{Re} \operatorname{Li}_{3}\left(\frac{1+i}{2}\right)=\frac{1}{48} \ln ^{3} 2-\frac{5}{192} \pi^{2} \ln 2+\frac{35}{64} \zeta(3)
$$

and $(c)$

$$
\begin{aligned}
8 \sum_{j=0}^{\infty} \frac{1}{16^{j}} & {\left[\frac{4}{(8 j+1)^{3}}-\frac{2}{(8 j+4)^{3}}-\frac{1}{(8 j+5)^{3}}-\frac{1}{(8 j+6)^{3}}\right] } \\
= & -\frac{\pi^{2}}{2} \ln 2+14 \zeta(3)+32 \operatorname{Im~Li}_{3}\left(\frac{1+i}{2}\right)
\end{aligned}
$$

Equation (2.35) is based upon the integral

$$
-4 \int_{0}^{1} \frac{(y-1) \ln (y / \sqrt{2}) d y}{y^{4}-2 y^{3}+4 y-4}=G+\frac{\pi^{2}}{32}
$$

and the summatory relation

$$
\sum_{j=0}^{\infty} \frac{1}{16^{j}} \frac{1}{(8 j+k)^{2}}+\frac{\ln 2}{2} \sum_{j=0}^{\infty} \frac{1}{16^{j}} \frac{1}{(8 j+k)}=-2^{k / 2} \int_{0}^{1 / \sqrt{2}} \frac{x^{k-1}}{1-x^{8}} \ln x d x,
$$

and turns out to be a combination of two known BBP-type formulas given in Section 5 of Ref. [2].

Lemma 4 parts (b) and (c) are constructed starting from the relation

$$
2^{k / 2} \int_{0}^{1 / \sqrt{2}} \frac{x^{k-1}}{1-x^{8}} \ln ^{2} x d x=\frac{1}{4} \sum_{j=0}^{\infty} \frac{1}{16^{j}} \frac{1}{(8 j+k)}\left[\ln ^{2} 2+\frac{4 \ln 2}{8 j+k}+\frac{8}{(8 j+k)^{2}}\right]
$$


We then form the sums

$$
\begin{gathered}
8 \sum_{j=0}^{\infty} \frac{1}{16^{j}}\left[\frac{4}{(8 j+1)^{3}}-\frac{2}{(8 j+4)^{3}}-\frac{1}{(8 j+5)^{3}}-\frac{1}{(8 j+6)^{3}}\right] \\
+4 \ln 2 \sum_{j=0}^{\infty} \frac{1}{16^{j}}\left[\frac{4}{(8 j+1)^{2}}-\frac{2}{(8 j+4)^{2}}-\frac{1}{(8 j+5)^{2}}-\frac{1}{(8 j+6)^{2}}\right] \\
+\ln ^{2} 2 \sum_{j=0}^{\infty} \frac{1}{16^{j}}\left[\frac{4}{(8 j+1)}-\frac{2}{(8 j+4)}-\frac{1}{(8 j+5)}-\frac{1}{(8 j+6)}\right] \\
=64 \int_{0}^{1} \frac{(y-1) \ln ^{2}(y / \sqrt{2}) d y}{y^{4}-2 y^{3}+4 y-4} \\
=16 G \ln 2-\pi \ln ^{2} 2+\frac{2}{3} i \ln ^{3} 2-\frac{5}{6} i \pi^{2} \ln 2-32 i \operatorname{Li}_{3}\left(\frac{1+i}{2}\right)+14\left(1+\frac{5}{4} i\right) \zeta(3) .
\end{gathered}
$$

We then apply both the degree 2 binary BBP-type formula for $\pi$ and part (a) of the Lemma, thereby eliminating the appearance of the constant $16 G \ln 2-\pi \ln ^{2} 2$. We then take the real and imaginary parts of the resulting equation. Since the imaginary part must vanish, we obtain part (b), and the real part gives the formula of part (c).

In regards to Eqs. (2.36) and (2.37), degree 3 binary BBP-type formulas are known for the constants $\zeta(3), \ln ^{3} 2, \pi \ln ^{2}, \pi^{2} \ln 2$, and $\pi^{3}$. However Lemma 4(c) does not appear in the compendium [2]. By means of Landen's transformation for $\mathrm{Li}_{3}$ or other functional relationships, the value $\operatorname{Li}_{3}[(1+i) / 2]$ may be related to other trilogarithm function values.

Lemma 4(b) and (c) may be computationally useful for providing a spigot algorithm for the constant $\operatorname{Li}_{3}[(1+i) / 2]$. We note that we have the alternative expressions

$$
\operatorname{Re} \operatorname{Li}_{3}\left(\frac{1+i}{2}\right)=\sum_{n=1}^{\infty} \sum_{m=0}^{n}\left[\left(\begin{array}{c}
n \\
4 m+4
\end{array}\right)-\left(\begin{array}{c}
n \\
4 m+2
\end{array}\right)\right] \frac{1}{2^{n} n^{3}},
$$




$$
\operatorname{Im} \operatorname{Li}_{3}\left(\frac{1+i}{2}\right)=\sum_{n=1}^{\infty} \sum_{m=0}^{n}\left[\left(\begin{array}{c}
n \\
4 m+1
\end{array}\right)-\left(\begin{array}{c}
n \\
4 m+3
\end{array}\right)\right] \frac{1}{2^{n} n^{3}}
$$

and that it may be possible to reach Eqs. (2.36) and (2.37) from them.

We mention an extension of Lemma 4 for BBP-type formulas for other polylogarithmic constants. These may be developed by using the integrals

$$
J_{n}=\frac{2^{n+1}}{n} \int_{0}^{1} \frac{(y-1) \ln ^{n}(y / \sqrt{2}) d y}{y^{4}-2 y^{3}+4 y-4}
$$

and

$$
\int_{0}^{\frac{1}{\sqrt{2}}} x^{k-1+8 j} \ln ^{n} x d x=\frac{(-1)^{n}}{(8 j+k)^{n+1}} \Gamma\left[n+1, \frac{1}{2}(8 j+k) \ln 2\right],
$$

together with the property given in Eq. (3.8) for the incomplete Gamma function. The evaluation of the integrals of Eq. (2.42) includes the constants $G \ln ^{n-1} 2, \pi^{j} \ln ^{k} 2$, where $j+k=n+1, \operatorname{Li}_{n}[(1 \pm i) / 2], \ln ^{p} 2 \operatorname{Li}_{q}(1 / 2)$, with $p+q=n+1, \operatorname{Li}_{n}[(1 \pm i) / 2]$, and $\ln ^{n-2} 2 \zeta(3)$.

Remark on sums of sine values. Supplementary to Eq. (2.7) we have the sum

$$
\sin \frac{x \pi}{10}+\sin \frac{3 x \pi}{10}+\sin \frac{7 x \pi}{10}+\sin \frac{9 x \pi}{10}=\{ \pm \sqrt{5}, 0\}
$$

for $x=1, \ldots, 20$, the - sign holds on the right side for $x=11, \ldots, 20$, and the result is zero when $(x, 10) \neq 1$, i.e., when $x$ is not co-prime with 10 . Here and within the rest of this remark the notation on the right side of the equation indicates that the expression on the left takes the values within the set indicated. Furthermore, we have the sine sums

$$
\sin \frac{x \pi}{12}+\sin \frac{5 x \pi}{12}+\sin \frac{7 x \pi}{12}+\sin \frac{11 x \pi}{12}=\{ \pm \sqrt{6}, 0\}
$$


for $x=1, \ldots, 24$ and the result is zero when $(x, 12) \neq 1$,

$$
\sin \frac{2 x \pi}{11}-\sin \frac{4 x \pi}{11}+\sin \frac{6 x \pi}{11}+\sin \frac{8 x \pi}{11}+\sin \frac{10 x \pi}{11}=\left\{ \pm \frac{\sqrt{11}}{2}, 0\right\}
$$

for $x=1, \ldots, 22$ and the result is zero when $x \equiv 0(\bmod 11)$,

$$
\sin \frac{2 x \pi}{15}+\sin \frac{4 x \pi}{15}+\sin \frac{8 x \pi}{15}+\sin \frac{14 x \pi}{15}=\left\{ \pm \frac{\sqrt{15}}{2}, 0\right\},
$$

for $x=1, \ldots, 15$ and the result is zero when $(x, 15) \neq 1$,

$$
\sin \frac{x \pi}{5}+\sin \frac{2 x \pi}{5}+\sin \frac{3 x \pi}{5}+\sin \frac{4 x \pi}{5}=\{ \pm \sqrt{5 \pm 2 \sqrt{5}}, 0\}
$$

for $x=1, \ldots, 10$ and the result is zero when $x=2,4,5,6,8$, and 10 ,

$$
\sin \frac{x \pi}{5}-\sin \frac{2 x \pi}{5}-\sin \frac{3 x \pi}{5}+\sin \frac{4 x \pi}{5}=\{\mp \sqrt{5 \mp 2 \sqrt{5}}, 0\},
$$

for $x=1, \ldots, 10$ and the result is zero when $x=2,4,5,6,8$, and 10 ,

$$
\sin \frac{x \pi}{8}+\sin \frac{3 x \pi}{8}+\sin \frac{7 x \pi}{8}=\left\{ \pm \frac{1}{2} \sqrt{10 \pm \sqrt{2}}, \frac{1}{\sqrt{2}},-1,0\right\},
$$

for $x=1, \ldots, 16$ and the result is zero when $x \equiv 0(\bmod 8)$, and

$$
\sin \frac{x \pi}{8}+\sin \frac{5 x \pi}{8}+\sin \frac{7 x \pi}{8}=\left\{ \pm \frac{1}{2} \sqrt{10 \pm \sqrt{2}},-\frac{1}{\sqrt{2}}, 1,0\right\},
$$

for $x=1, \ldots, 16$ and the result is zero when $x \equiv 0(\bmod 8)$. We omit the values taken by a multitude of other sine combinations, including $\sin \frac{x \pi}{8} \pm \sin \frac{7 x \pi}{8}, \sin \frac{x \pi}{8} \pm \sin \frac{5 x \pi}{8}$, and $\sin \frac{3 x \pi}{8}+\sin \frac{7 x \pi}{8}$. Many of the combinations of sine values listed in this Remark are related to factorizations of Chebyshev polynomials. Such sums as given have implications for relations between Clausen function values. We return to this topic in the Discussion section. 


\section{Proof of Proposition 1}

Rather than restrict attention to $I_{7}$, we consider the more general integrals

$$
I(n) \equiv \int_{\pi / 3}^{\pi / 2} \ln ^{n}\left|\frac{\tan t+\sqrt{7}}{\tan t-\sqrt{7}}\right| d t=\int_{\sqrt{3}}^{\infty} \ln ^{n}\left|\frac{u+\sqrt{7}}{u-\sqrt{7}}\right| \frac{d u}{1+u^{2}} .
$$

By splitting the integral and performing further changes of variable we have

$$
\begin{aligned}
I(n)=I^{(1)}(n) & +I^{(2)}(n)=\int_{\sqrt{3}}^{\sqrt{7}} \ln ^{n}\left(\frac{\sqrt{7}+u}{\sqrt{7}-u}\right) \frac{d u}{1+u^{2}}+\int_{\sqrt{7}}^{\infty} \ln ^{n}\left(\frac{u+\sqrt{7}}{u-\sqrt{7}}\right) \frac{d u}{1+u^{2}} \\
& =\frac{\sqrt{7}}{2}\left[\int_{r_{73}}^{\infty} \frac{\ln ^{n} v d v}{\left(2 v^{2}-3 v+2\right)}+\int_{1}^{\infty} \frac{\ln ^{n} v d v}{\left(2 v^{2}+3 v+2\right)}\right] .
\end{aligned}
$$

By the use of Lemma 2(a), from the form given in Eq. (3.2), it is evident that

$$
I^{(2)}(1)=\mathrm{Cl}_{2}\left[\cos ^{-1}(-3 / 4)\right]=\mathrm{Cl}_{2}\left(\pi+\theta_{-}\right)=-\mathrm{Cl}_{2}\left(\pi+\theta_{+}\right) .
$$

We have

$$
\begin{gathered}
\frac{I^{(1)}(n)}{2 \sqrt{7}}=\frac{1}{4} \int_{r_{73}}^{\infty} \frac{\ln ^{n} v d v}{\left(2 v^{2}-3 v+2\right)}=\frac{1}{8} \int_{r_{73}}^{\infty} \frac{\ln ^{n} v d v}{\left(v-v_{+}\right)\left(v-v_{-}\right)} \\
=\frac{1}{8\left(v_{+}-v_{-}\right)} \int_{r_{73}}^{\infty}\left[\frac{1}{v-v_{+}}-\frac{1}{v-v_{-}}\right] \ln ^{n} v d v,
\end{gathered}
$$

where $v_{ \pm}=(3 \pm i \sqrt{7}) / 4=\exp \left(i \theta_{ \pm}\right)$. By making the change of variable $y=1 / v$ and expanding the bracketed terms in geometric series, we have

$$
\frac{I^{(1)}(n)}{2 \sqrt{7}}=\frac{(-1)^{n}}{8\left(v_{+}-v_{-}\right)} \int_{0}^{1 / r_{73}} \ln ^{n} y \sum_{\ell=0}^{\infty}\left(\frac{1}{v_{-}^{\ell+1}}-\frac{1}{v_{+}^{\ell+1}}\right) \frac{d y}{y^{\ell+2}} .
$$

We perform the integral in terms of the incomplete Gamma function $\Gamma(x, y)$, finding that

$$
\frac{I^{(1)}(n)}{2 \sqrt{7}}=\frac{(-1)^{n+1}}{8\left(v_{+}-v_{-}\right)} \sum_{\ell=0}^{\infty}\left(\frac{1}{v_{-}^{\ell+1}}-\frac{1}{v_{+}^{\ell+1}}\right) \frac{\Gamma\left[n+1,-(\ell+1) \ln r_{73}\right]}{(\ell+1)^{n+1}} .
$$


We next use the property for $n \geq 0$ an integer

$$
\Gamma(n+1, x)=n ! e^{-x} \sum_{m=0}^{n} \frac{x^{m}}{m !}
$$

to write

$$
\frac{I^{(1)}(n)}{2 \sqrt{7}}=\frac{(-1)^{n+1} n !}{8\left(v_{+}-v_{-}\right)} \sum_{\ell=1}^{\infty}\left(\frac{1}{v_{-}^{\ell}}-\frac{1}{v_{+}^{\ell}}\right) \frac{r_{73}^{\ell}}{\ell^{n+1}} \sum_{m=0}^{n} \frac{\ell^{m}}{m !} \ln ^{m}\left(\frac{1}{r_{73}}\right) .
$$

Since $v_{+}$and $v_{-}$are complex conjugates, the sum over $\ell$ in this equation is pure imaginary, as is $v_{+}-v_{-}=i \sqrt{7} / 2$.

We now specialize to $n=1$, whereby

$$
\begin{aligned}
& \frac{I^{(1)}(1)}{2 \sqrt{7}}=\frac{1}{8\left(v_{+}-v_{-}\right)}\left[\sum_{\ell=1}^{\infty}\left(\frac{1}{v_{-}^{\ell}}-\frac{1}{v_{+}^{\ell}}\right) \frac{r_{73}^{\ell}}{\ell^{2}}-\ln r_{73} \sum_{\ell=1}^{\infty}\left(\frac{1}{v_{-}^{\ell}}-\frac{1}{v_{+}^{\ell}}\right) \frac{r_{73}^{\ell}}{\ell}\right] \\
& =\frac{1}{8\left(v_{+}-v_{-}\right)}\left[\operatorname{Li}_{2}\left(\frac{r_{73}}{v_{-}}\right)-\operatorname{Li}_{2}\left(\frac{r_{73}}{v_{+}}\right)-\ln r_{73} \ln \left(\frac{1-v_{-} r_{73}}{1-v_{+} r_{73}}\right)\right],
\end{aligned}
$$

where we used the defining series form of $\mathrm{Li}_{2}$, and the fact that $v_{-} v_{+}=1$. The latter logarithmic value may be rewritten as

$$
\ln \left(\frac{1-v_{-} r_{73}}{1-v_{+} r_{73}}\right)=-2 i \tan ^{-1} \frac{1}{5}(2 \sqrt{3}+\sqrt{7})=2 i \omega_{+},
$$

and we now use relation (1.15), taking advantage of the fact that the right side of Eq. (3.10) is the ratio of two pure imaginary quantities.

We then find that

$$
\frac{I^{(1)}(1)}{2 \sqrt{7}}=\frac{1}{4 \sqrt{7}}\left[\mathrm{Cl}_{2}\left(2 \omega_{+}\right)-\mathrm{Cl}_{2}\left(2 \omega_{+}+2 \theta_{+}\right)+\mathrm{Cl}_{2}\left(2 \theta_{+}\right)\right],
$$

where we repeatedly made use of $\omega_{+}+\omega_{-}=0$. We combine with the result in Eq. (3.4) for $I^{(2)}(1)$ and use the duplication formula (1.17), giving Eq. (1.13). 


\section{Discussion and additional results}

Since our result for $I_{7}$ involves angles other than $\theta_{7}=2 \tan ^{-1} \sqrt{7}$ of Eq. (26) of Ref. [5], another Clausen function value relation is suggested. In fact, by Eqs. (4.61) and (4.63) of [12] we have the relation

$$
2 \mathrm{Cl}_{2}\left(2 \theta_{+}\right)-3 \mathrm{Cl}_{2}\left(2 \theta_{+}-\theta_{7}\right)-\mathrm{Cl}_{2}\left(3 \theta_{7}-2 \theta_{+}\right)+6 \mathrm{Cl}_{2}\left(\pi+\theta_{7}\right)=0
$$

Since the duplication formula (1.17) holds, the value $\mathrm{Cl}_{2}\left(\theta_{+}\right)$is then expressible as a combination of the Clausen function at other angles. Additionally, as we have noted in Eq. (1.12b), we have $2 \omega_{+}=\theta_{7}-4 \pi / 3$.

The sine relations given at the end of Section 2 lead to a series of other Clausen function and hence Hurwitz zeta and polygamma function expressions. For $2 q$ even, let

$$
\mathrm{Cl}_{2 q}(\theta) \equiv \sum_{n=1}^{\infty} \frac{\sin n \theta}{n^{2 q}}
$$

be the generalized Clausen function. Then, for instance, from Eq. (2.7) we have

$$
\begin{gathered}
\mathrm{Cl}_{q}\left(\frac{2 \pi}{7}\right)+\mathrm{Cl}_{q}\left(\frac{4 \pi}{7}\right)-\mathrm{Cl}_{q}\left(\frac{6 \pi}{7}\right) \\
=\frac{\sqrt{7}}{2} 7^{-q}\left[\zeta\left(q, \frac{1}{7}\right)+\zeta\left(q, \frac{2}{7}\right)-\zeta\left(q, \frac{3}{7}\right)+\zeta\left(q, \frac{4}{7}\right)-\zeta\left(q, \frac{5}{7}\right)-\zeta\left(q, \frac{6}{7}\right)\right],
\end{gathered}
$$

that presents an extension of Lemma 1. We omit related expressions in terms of the polygamma functions, as well as similar expressions coming from additional sine function value relations.

The method of proof of Proposition 1 has furnished the more general result for the integrals $I^{(1)}(n)$ in terms of polylogarithm functions $\mathrm{Li}_{j}$ and hence in terms of 
generalized Clausen functions. From Eq. (3.9) we have

$$
\begin{gathered}
I^{(1)}(n)=(-1)^{n} \frac{i}{2}\left\{\operatorname{Li}_{n+1}\left(\frac{r_{73}}{v_{-}}\right)-\operatorname{Li}_{n+1}\left(\frac{r_{73}}{v_{+}}\right)+\operatorname{Li}_{n}\left(\frac{r_{73}}{v_{-}}\right)-\operatorname{Li}_{n}\left(\frac{r_{73}}{v_{+}}\right)+\ldots\right. \\
+\frac{\ln ^{j}\left(1 / r_{73}\right)}{j !}\left[\operatorname{Li}_{n-j+1}\left(\frac{r_{73}}{v_{-}}\right)-\operatorname{Li}_{n-j+1}\left(\frac{r_{73}}{v_{+}}\right)\right]+\ldots \\
\left.+\frac{\ln ^{n-1}\left(1 / r_{73}\right)}{(n-1) !}\left[\operatorname{Li}_{2}\left(\frac{r_{73}}{v_{-}}\right)-\operatorname{Li}_{2}\left(\frac{r_{73}}{v_{+}}\right)\right]\right\}+(-1)^{n} \frac{i}{2} \ln ^{n}\left(1 / r_{73}\right) \ln \left(\frac{1-v_{-} r_{73}}{1-v_{+} r_{73}}\right) .
\end{gathered}
$$

Supplementing Eq. (4.2), one writes for $2 r+1$ an odd integer

$$
\mathrm{Cl}_{2 r+1}(\theta) \equiv \sum_{n=1}^{\infty} \frac{\cos n \theta}{n^{2 r+1}}
$$

Similarly, the integrals $I^{(2)}(n)$ and $I(n)$ may be evaluated.

Also following the method of Proposition 1 we may make the following extension of Eq. (1.8). Let $\theta_{+}=-\tan ^{-1}\left(\sqrt{1-b^{2}} / b\right)$ and $\omega_{+}=\tan ^{-1}\left[\sqrt{1-b^{2}} /(a+b)\right]$. Then we have

Proposition 2. For real $a \geq 0$ and $|b|<1$ we have

$$
\begin{gathered}
I(a, b) \equiv \int_{a}^{\infty} \frac{\ln y d y}{y^{2}+2 b y+1}=\frac{1}{2} \frac{1}{\sqrt{1-b^{2}}}\left[\mathrm{Cl}_{2}\left(2 \omega_{+}\right)-\mathrm{Cl}_{2}\left(2 \omega_{+}+2 \theta_{+}\right)+\mathrm{Cl}_{2}\left(2 \theta_{+}\right)\right] \\
=\frac{1}{2} \frac{1}{\sqrt{1-b^{2}}}\left[\mathrm{Cl}_{2}\left(2 \theta_{2}-2 \theta_{1}\right)-\mathrm{Cl}_{2}\left(\pi-2 \theta_{1}\right)+\mathrm{Cl}_{2}\left(\pi-2 \theta_{2}\right)\right]
\end{gathered}
$$

where $\tan \theta_{1}=b / \sqrt{1-b^{2}}$ and $\tan \theta_{2}=(1 / a+b) / \sqrt{1-b^{2}}$.

In obtaining Eq. (4.6), we write

$$
I(a, b)=-\frac{1}{\left(y_{+}-y_{-}\right)} \int_{0}^{1 / a} \ln v\left[\frac{1}{\left(1-y_{+} v\right)}-\frac{1}{\left(1-y_{-} v\right)}\right] \frac{d v}{v},
$$

where $y_{ \pm} \equiv-b \pm i \sqrt{1-b^{2}}=\exp \left(i \theta_{ \pm}\right)$. We then proceed similarly as in the proof of Proposition 1. Of course Eq. (4.6) may be rewritten with the aid of the duplication formula (1.17). 
For Eq. (4.7), we integrate an equality with respect to $a$ and then determine the constant of integration that may depend only upon $b$. By the use of the chain rule, the relation

$$
\frac{d \theta_{2}}{d a}=-\frac{\sqrt{1-b^{2}}}{a^{2}+2 a b+1},
$$

and the first expression on the right side of Eq. (1.14) for $\mathrm{Cl}_{2}$, we easily verify the equality of both sides of Eq. (4.7) upon differentiating with respect to $a$. The constant of integration may be found at $a=1$. By Lemma 2(a) [cf. Eq. (2.11) with $n=1$ ] we have $I(1, \pm b)=\mathrm{Cl}_{2}\left(\cos ^{-1} b\right) / \sqrt{1-b^{2}}$.

Having determined the integral $I(a, b)$ by two different methods, we have found a relation among Clausen function values.

Corollary 2. With $\theta_{+}, \omega_{+}, \theta_{1}$, and $\theta_{2}$ as in Proposition 2, we have

$\mathrm{Cl}_{2}\left(2 \omega_{+}\right)-\mathrm{Cl}_{2}\left(2 \omega_{+}+2 \theta_{+}\right)+\mathrm{Cl}_{2}\left(2 \theta_{+}\right)=\mathrm{Cl}_{2}\left(2 \theta_{2}-2 \theta_{1}\right)-\mathrm{Cl}_{2}\left(\pi-2 \theta_{1}\right)+\mathrm{Cl}_{2}\left(\pi-2 \theta_{2}\right)$.

This relation seems to reflect the property $\tan ^{-1} x+\cot ^{-1} x=\tan ^{-1} x+\tan ^{-1}(1 / x)=$ $\pi / 2$ for $x>0$.

From Proposition 2 we recover many known special cases. In particular, we have $I(0, b)=0$, when $\omega_{+}=-\theta_{+}$. In turn, we have

Corollary 3. We have for $c>0$ and $0<t<\pi$

$$
\int_{0}^{\infty} \frac{\ln x d x}{x^{2}+2 x c \cos t+c^{2}}=\frac{\ln c}{c} \frac{t}{\sin t},
$$

recovering a formula of Ref. [9] (p. 533). In order to obtain Eq. (4.11) we use the scaling substitution $y=x / c$ and make use of the fact that $I(0, b)=0$. 
Due to the presence of the parameter $b$ in Proposition 2, we may obtain expressions for integrals of the form

$$
\int_{a}^{\infty} \frac{y^{j} \ln y d y}{\left(y^{2}+2 b y+1\right)^{j+1}}=\frac{(-1)^{j}}{2^{j} j !} \frac{\partial^{j} I(a, b)}{\partial b^{j}}
$$

\section{Acknowledgement}

This work was partially supported by Air Force contract number FA8750-06-10001. 


\section{References}

[1] M. Abramowitz and I. A. Stegun, Handbook of Mathematical Functions, National Bureau of Standards (1972).

[2] D. H. Bailey, A compendium of BBP-type formulas for mathematical constants, http://crd.lbl.gov/ dhbailey/dhbpapers/bbp-formulas.pdf (2004).

[3] D. H. Bailey et al., Experimental Mathematics in Action, A. K. Peters, Wellesley, MA (2007).

[4] D. H. Bailey and J. M. Borwein, Computer-assisted discovery and proof, preprint http://crd.lbl.gov/ dhbailey/dhbpapers/comp-disc-proof.pdf (2007).

[5] J. M. Borwein and D. J. Broadhurst, Determination of rational Dedekindzeta invariants of hyperbolic manifolds and Feynman knots and links, arxiv:hep-th/9811173 (1998).

[6] J. M. Borwein, I. J. Zucker, and J. Boersma, The evaluation of character Euler double sums, to appear in Ramanujan J. (2007).

[7] D. J. Broadhurst, Massive 3-loop Feynman diagrams reducible to $\mathrm{SC}^{*}$ primitives of algebras of the sixth root of unity, Eur. Phys. J. C 8, 311-333 (1999).

[8] P. J. de Doelder, On the Clausen integral $\mathrm{Cl}_{2}(\theta)$ and a related integral, J. Comput. Appl. Math. 11, 325-330 (1984). 
[9] I. S. Gradshteyn and I. M. Ryzhik, Table of Integrals, Series, and Products, Academic Press, New York (1980).

[10] C. C. Grosjean, Formulae concerning the computation of the Clausen integral $\mathrm{Cl}_{2}(\theta)$, J. Comput. Appl. Math. 11, 331-342 (1984).

[11] E. R. Hansen, A table of series and products, Prentice Hall (1975).

[12] L. Lewin, Polylogarithms and associated functions, North Holland (1981).

[13] L. Lewin, ed., Structural properties of polylogarithms, American Mathematical Society (1991).

[14] F. A. Lunev, Evaluation of two-loop self-energy diagram with three propagators, Phys. Rev. D 50, 7735-7737 (1994). It appears that in Eq. (18), the integrand factor $(1-\lambda y)$ should read $(1-\lambda x)$.

[15] L. C. Maximon, The dilogarithm function for complex argument, Proc. Royal Soc. A 459, 2807-2819 (2003). 